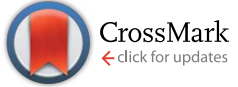

Cite this: RSC Adv., 2017, 7, 13947

Received 1st December 2016 Accepted 17th February 2017

DOI: $10.1039 / c 6 r a 27608 c$

rsc.li/rsc-advances

\section{Inhibitory effect of hydrophobic fullerenes on the $\beta$-sheet-rich oligomers of a hydrophilic GNNQQNY peptide revealed by atomistic simulations $\uparrow$}

\begin{abstract}
Jiangtao Lei, Ruxi Qi, Luogang Xie, Wenhui Xi and Guanghong Wei*
Increasing numbers of experiments show that carbon nanoparticles can significantly influence protein fibrillation, a process associated with many human amyloidosis. Our previous studies demonstrate that fullerenes and carbon nanotubes can markedly inhibit the formation of $\beta$-sheet-rich oligomers and amyloid fibrils of the hydrophobic KLVFFAE peptide, the central hydrophobic core fragment of Alzheimer's amyloid- $\beta$ protein, mostly through hydrophobic and aromatic-stacking interactions. In this study, the effect of hydrophobic fullerene $C_{60}$ on the oligomeric structures of the hydrophilic GNNQQNY peptide, the key amyloid-forming fragment of yeast prion protein Sup35, is investigated by extensive replica exchange molecular dynamics simulations. Our simulations, starting from eight random chains, show that fullerene can greatly impede the $\beta$-sheet formation of the GNNQQNY peptide and induce oligomer shifting from various $\beta$-sheet-rich structures to disordered coil conformations. Strikingly, fullerenes completely prevent fibril-like bilayer $\beta$-sheets, formed by GNNQQNY peptides alone. We find that $C_{60}$ molecules interact strongly with the nonpolar aliphatic groups of polar residues N3, Q4 and Q5 and increase the water exposure of the peptide backbone, thus block the inter-peptide N3-Q4, Q4-Q4 and Q4-Q5 interactions which are crucial for oligomerization and $\beta$-sheet formation. These results, together with our previous studies on the KLVFFAE peptide, suggest that hydrophobic fullerenes could inhibit the aggregation of both hydrophobic and hydrophilic peptides. This finding may offer new clues for the design of therapeutic agents against different types of amyloidosis.
\end{abstract}

\section{Introduction}

Many proteins have an intrinsic tendency to aggregate into amyloid deposits that are hallmarks of numerous fatal diseases such as Alzheimer's, Parkinson's, Huntington's and CreutzfeldtJakob diseases..$^{1-3}$ In spite of different protein composition, the main components of these amyloid deposits are amyloid fibrils that display a common cross- $\beta$ structure with the $\beta$-strands perpendicular to and the inter-strand hydrogen bonds parallel to the fibril axis. ${ }^{4}$ This finding indicates that a general principle may govern amyloid fibril formation. ${ }^{5}$ Most importantly, increasing evidence suggests that small oligomers formed in the early stage of aggregation, rather than the fibrils themselves, are the most cytotoxic species in neurodegenerative diseases. ${ }^{6,7}$ However, due to the transient nature of oligomers, their atomic-level structures and the molecular mechanisms of formation, toxicity, and inhibition are still not fully understood. ${ }^{8}$ Numerous experimental studies demonstrated that hydrophobic fragments (such as

State Key Laboratory of Surface Physics, Key Laboratory for Computational Physical Sciences (Ministry of Education), Department of Physics, Fudan University, 220 Handan Road, Shanghai, 200433, China.E-mail: ghwei@fudan.edu.cn

$\dagger$ Electronic supplementary information (ESI) available. See DOI: $10.1039 / \mathrm{c} 6 \mathrm{ra} 27608 \mathrm{c}$
KLVFFAE in the full length amyloid- $\beta$ (A $\beta),{ }^{9}$ VQIVYK in tau $\operatorname{protein}^{\mathbf{1 0}}$ and GVIGIAQ in superoxide dismutase (SOD1) ${ }^{1 \mathbf{1 1}}$ ) are usually the important triggers for the fibrillation of full-length proteins, while polar segments (such as GNNQQNY in yeast prion Sup35 protein ${ }^{12}$ and polyglutamine sequence in Huntingtin protein $^{13}$ ) also play key roles on protein aggregation. Most interestingly, these segments themselves can also form amyloid fibrils that displays many of the common characteristics as the fibrils formed by the full-length proteins..$^{9-12,14}$ Among them, GNNQQNY peptide serves as a good model for studying the early stage of aggregation due to its availability of the atomic fibril-like structure determined by Eisenberg et al. using X-ray microcrystallography. ${ }^{15}$ The fibril-like structure from the GNNQQNY microcrystal contains a double $\beta$-sheet, with each sheet formed by main chain hydrogen bonds, and a dry, tightly selfcomplementing steric zipper formed by side chains protruding from the sheets. ${ }^{15}$ Solid state NMR experiments indicate significant structural differences between the structures of crystalline and fibrillary forms of this peptide, with a clearly increased complexity in the fibril structure. ${ }^{16-19}$ Very recently, two forms of high-resolution structures of GNNQQNY nanocrystals were obtained by Eisenberg et al. using cryo-EM method ${ }^{\mathbf{2 0}}$ and they display different space groups: $P 2_{1}$ and $P 2_{1} 2_{1} 2_{1}$. 
Complementary to experiments, molecular dynamics (MD) simulations can provide the oligomerization dynamics and thermodynamics of GNNQQNY peptides at the atomic level. By using coarse-grained or atomistic MD simulations, several research groups investigated the structural stability of different sizes of preformed fibril-like oligomers, ${ }^{21-23}$ the oligomerization dynamics/kinetics of the peptide ${ }^{24-31}$ the growth and assembly dynamics of preformed protofibrils, ${ }^{32,33}$ and the energetics and thermodynamics of oligomers (from dimers to hexamers). ${ }^{\mathbf{3 4 - 3 6}}$ Two common findings from these studies are: (1) for preformed fibril-like oligomers, interstrand backbone-backbone and side chain-side chain hydrogen bonds are the main forces to stabilize a $\beta$-sheet, and steric zipper interactions between the side chains of N2, Q4, and N6 in two different sheets hold the sheets together; (2) irrespective of the force field used, antiparallel $\beta$ sheet dimers and trimers are populated in the early stage of aggregation, although $\beta$-strands in GNNQQNY fibrils are in parallel alignment. ${ }^{15,20}$ These computational studies have enhanced our understanding of the physical forces stabilizing the preformed fibrils and the formation pathway of small oligomers. However, the conformational ensembles of oligomers larger than hexamers remain to be determined at the atomic level.

Finding effective inhibitors that prevent the formation of toxic $\beta$-sheet-rich aggregates and revealing the inhibitory mechanism are important for the development of therapeutic agents. Researchers have found that nanoparticles (such as polymeric nanoparticles, ${ }^{37}$ gold nanoparticles ${ }^{38}$ and carbon nanoparticles ${ }^{39}$ ), peptides, ${ }^{\mathbf{4 0 , 4 1}}$ small molecules, ${ }^{\mathbf{4 1 - 4 3}}$ and antibodies ${ }^{\mathbf{4 1}}$ can significantly interfere with the aggregation process of proteins. Among them, carbon nanoparticles (including fullerenes, carbon nanotubes, and graphene oxides) have attracted considerable attention, ${ }^{39,44,45}$ due to their good biocompatibility, low cytotoxicity and high capacity to cross blood brain barriers. ${ }^{46-48}$ Fullerene $\mathrm{C}_{60}$ with its $0.7 \mathrm{~nm}$ wide cage, presents the suitable size and shape for the formation of stable complexes with proteins. ${ }^{49}$ The presence of both five and six-membered rings in $\mathrm{C}_{60}$ (one $\mathrm{C}_{60}$ molecule containing 12 pentagons and 20 hexagons) suggests strong fullereneprotein interaction through aromatic stackings. ${ }^{\mathbf{5 0}}$ Increasing experimental studies have demonstrated that fullerene $\mathrm{C}_{60}$ and its derivatives have remarkable anti-amyloid capacity and neuroprotective potentials. ${ }^{51-57}$ On the computational side, it has been reported that fullerene inhibits protein fibrillation or disrupts preformed fibrils by mostly binding to regions consisting of hydrophobic or aromatic residues. ${ }^{58-61}$ In particular, our recent work suggests that fullerenes prevent the fibrillation of hydrophobic KLVFFAE (A $\beta(16-22))$ peptide (the central hydrophobic core fragment of $A \beta$ protein) by inhibiting the formation of $\beta$-sheet oligomers. ${ }^{50}$ However, the effect of the fullerene $\mathrm{C}_{60}$ on the aggregation of hydrophilic peptides remains elusive.

In this work, as a first step to understand the influence of hydrophobic fullerene on the aggregation of hydrophilic peptides, we investigated the conformational ensemble of GNNQQNY oligomers in the absence and presence of $\mathrm{C}_{60}$ molecules by performing extensive atomistic replica exchange molecular dynamics (REMD) simulations starting from multiple random peptide chains. Similar to our recent REMD study of $A \beta(16-22)$ system, ${ }^{50}$ eight GNNQQNY peptide chains and three $\mathrm{C}_{60}$ molecules were simulated in the GNNQQNY system. Our simulations show that in the absence of $\mathrm{C}_{60}$ molecules, GNNQQNY octamers adopt a diverse ensemble of conformations, including predominant disordered coil-rich states, low-populated disordered $\beta$-sheet-rich states and ordered bilayer $\beta$-sheets. However, in the presence of $\mathrm{C}_{60}, \beta$ sheet-rich conformations are greatly reduced, in particular, the ordered bilayer $\beta$-sheets are completely suppressed. Binding probability and binding energy analyses demonstrate that $\mathrm{C}_{60}$ interacts strongly with the nonpolar aliphatic groups of polar residues $\mathrm{N} 3$, Q4 and Q5, thus interferes with the inter-peptide N3-Q4, Q4-Q4 and Q4-Q5 interactions which are important for $\beta$-sheet formation and aggregation of GNNQQNY peptide. The role of water molecules on peptide-peptide interactions are also discussed.

\section{Models and methods}

\section{Initial conformations of GNN and $\mathrm{GNN}+\mathrm{C}_{60}$ systems}

We investigated two different systems: GNNQQNY octamer alone (GNN for short) and GNNQQNY octamer in the presence of three $\mathrm{C}_{60}$ molecules ( $\mathrm{GNN}+\mathrm{C}_{60}$ for short). The two termini of each GNNQQNY peptide chain are charged $\left(\mathrm{NH}_{3}{ }^{+}\right.$and $\left.\mathrm{COO}^{-}\right)$to mimic the neutral experimental $\mathrm{pH}$ condition. ${ }^{62}$ Each system consists of eight peptide chains with random coil conformation for each chain. The eight GNNQQNY chains were placed randomly in a $6.7 \times 6.7 \times 6.7 \mathrm{~nm}^{3}$ box filled with $\mathrm{SPC}$ water molecules. Three $\mathrm{C}_{60}$ molecules were randomly displaced in the peptide system. The peptide concentrations in the two systems are $36.9 \mathrm{mg} \mathrm{ml}^{-1}$, which is close to the experimental peptide concentration for GNNQQNY fibril formation. ${ }^{63}$ The starting states of the two systems are given in Fig. S1 (ESI $\dagger$ ).

\section{REMD simulations}

We conducted one 250 ns REMD $^{64}$ simulation for each system using the GROMACS-4.5.3 software package, ${ }^{65}$ following the procedure adopted for our previous REMD simulations. ${ }^{\mathbf{5 0 , 6 6 - 6 8}}$ The GROMOS96 $43 \mathrm{a} 1$ force field ${ }^{69}$ was used, in accordance with previous computational studies of GNNQQNY aggregation. ${ }^{22-24,26}$ As demonstrated by a number of computational studies, ${ }^{27,35,70-73}$ REMD method is useful for investigating the aggregation of a series of peptides such as GNNQQNY ${ }^{27,35}$ and KLVFFAE. $^{68}$ Moreover, our previous works on A $\beta(16-22)$ aggregation showed that the predictions from REMD simulations were confirmed by experiments. ${ }^{\mathbf{5 0 , 7 4}}$ Thus, we employed the REMD method to investigate the octameric structures of GNNQQNY peptide and the influence of $\mathrm{C}_{60}$ nanoparticles. The REMD simulations were performed in the NPT ensemble using 48 replicas (250 ns per replica) at temperatures ranging from $310 \mathrm{~K}$ to $411 \mathrm{~K}$. Electrostatic interactions were calculated using particle mesh Ewald algorithm ${ }^{75}$ with a real space cut-off of $1.0 \mathrm{~nm}$. van der Waals interactions were calculated using a cutoff of $1.4 \mathrm{~nm}$. Constrains were applied for bond lengths of peptides using Linear Constraint Solver (LINCS) algorithm ${ }^{76}$ and for those of water molecules using SHAKE algorithm, ${ }^{77}$ 
allowing an integration time step of 2 fs. The solute and solvent were separately coupled to external temperature and pressure baths using velocity rescaling ${ }^{78}$ and Parrinello-Rahman ${ }^{79}$ coupling methods. The temperature- and pressure-coupling constants are 0.1 and $1.0 \mathrm{ps}$, respectively. Conformations were saved every 2 ps. The swap time between two neighboring replicas is 2 ps. The average acceptance ratio is $\sim 21 \%$, as shown in Fig. S2 (ESI†).

\section{Analysis methods}

The DSSP program was used to calculate the secondary structure. The probability distribution of $\beta$-sheet size was calculated to characterize the structural feature of aggregated states. For an $i$-stranded $\beta$-sheet, the size of this $\beta$-sheet is the same as the number of $\beta$-strand in the sheet. For example, the $\beta$-sheet size of a three-stranded $\beta$-sheet is three. Two peptide chains are considered to form a $\beta$-sheet if (i) at least two consecutive residues in each chain visit the $\beta$-sheet state and (ii) the two chains form at least two backbone hydrogen bonds $(\mathrm{H}-$ bonds). ${ }^{68,80}$ One $\mathrm{H}$-bond is considered as formed if the $\mathrm{N} \cdots \mathrm{O}$ distance is less than $0.35 \mathrm{~nm}$ and the $\mathrm{N}-\mathrm{H} \cdots \mathrm{O}$ angle is greater than $150^{\circ}$. The percentage $P_{i}$ of the $\beta$-sheet with size $=i$ is calculated using the following formula.

$$
P_{i}=\frac{n_{i} \times i}{\sum_{i=1}^{N}\left(n_{i} \times i\right)}
$$

here, $n_{i}$ is the number of $\beta$-sheet with size $=i$ and $N$ is the total number of chains $(N=8)$. Following our previous work, ${ }^{\mathbf{6 8 , 8 0}}$ we used the connectivity length (CL) to characterize the structural orderness of the octamers. For each octamer, the CL is defined as the sum over the square root of the $\beta$-sheet size and the number of chains with a random coil conformation, i.e. $\mathrm{CL}=\sum_{i} \sqrt{i}$. For example, the $\mathrm{CL}$ of an octamer consisting of one four-stranded $\beta$-sheet and four random chains without $\beta$ sheets is $\sqrt{4}+4 \times \sqrt{1}=6$, and the CL of an octamer consisting of two four-stranded $\beta$-sheets is $\sqrt{4}+\sqrt{4}=4$. Thus, the larger the connectivity length is, the more disordered the octamer becomes. We also calculated the ratio of parallel/antiparallel $(\mathrm{P} /$ AP) alignments in a $\beta$-sheet. The two $\beta$-sheet-forming chains are considered to form an antiparallel (parallel) $\beta$-sheet if the scalar product of their end-to-end unit vector is less than -0.7 (greater than 0.7$)$.

Potential of mean force (PMF) was constructed using the relation - $R T \ln H(x, y)$, where $H(x, y)$ is the histogram of two selected reaction coordinates: the radius of gyration $\left(R_{\mathrm{g}}\right)$ of the octamer and the number of hydrogen bonds (number of $\mathrm{H}$ bonds). Here, the H-bond number denotes the total number of intra- and inter-peptide H-bonds. The Daura cluster analysis method ${ }^{81}$ was used to classify the conformations sampled in the REMD simulations with a $\mathrm{C}_{\alpha^{-}}$-root-mean-square-deviation $\left(\mathrm{C}_{\alpha^{-}}\right.$ RMSD) cutoff of $0.3 \mathrm{~nm}$. The inter-peptide interactions and the peptide- $\mathrm{C}_{60}$ interactions were estimated respectively by the contact probability of residue-residue and residue- $\mathrm{C}_{60}$ pairs. Here, a contact is defined when the aliphatic carbon atoms of two nonsequential side chains (or main chains) come within $0.54 \mathrm{~nm}$ or any other atoms of two nonsequential side chains (or main chains) lie within $0.46 \mathrm{~nm} .^{68}$

We estimated the binding free energy between each amino acid residue and $\mathrm{a}_{60}$ molecule using molecular mechanics/ linear Poisson-Boltzmann surface area (MM/PBSA) method implemented for the GROMACS package (g_mmpbsa tool) ${ }^{82}$ It is challenging to calculate the conformational entropy for systems with large conformational changes using MM/PBSA. ${ }^{\mathbf{8 2 , 8 3}}$ In this study, the binding free energy is the relative binding energy in which the molecular mechanics potential energy in a vacuum and the solvation free energy were included, while the contribution of conformational entropy was ignored, in accordance with a number of previous computational studies. ${ }^{\mathbf{4 3 , 5 0 , 6 1 , 8 4}}$ Namely, the binding free energy $\left(\Delta G_{\text {binding }}\right)$ was calculated as: $\Delta G_{\text {binding }}=\Delta E_{\mathrm{MM}}+\Delta G_{\text {sol }}$. The $\Delta E_{\mathrm{MM}}$ is the potential energy in vacuum and $\Delta G_{\text {sol }}\left(=\Delta G_{\text {polar }}+\Delta G_{\text {nonpolar }}\right)$ is the solvation free energy that is required to transfer a solute from vacuum into the solvent. The $\Delta G_{\text {polar }}$ and $\Delta G_{\text {nonpolar }}$ are respectively the polar and non-polar contributions to the solvation free energy. The $\Delta G_{\text {polar }}$ was calculated by the PB model and the $G_{\text {nonpolar was }}$ estimated by the solvent accessible surface area. For comparison, we also calculated the contact probability between each residue and $\mathrm{C}_{60}$. The VMD program ${ }^{85}$ was used for trajectory visualization and for graphical structure analysis.

\section{Results and discussion}

\section{Convergence check of the REMD simulations}

We examined the convergence of the two 250 ns REMD simulations by comparing the following parameters within two different time intervals using the 100-175 ns and 175-250 ns data at $310 \mathrm{~K}$ for the two systems. As shown in Fig. S3, $\uparrow$ the probabilities of dominant secondary structure (coil, $\beta$-sheet and $\beta$-bridge) of each residue are quite similar between the two time periods. The distribution of total number of $\mathrm{H}$-bonds and that of side chain contact number overlap very well within the two independent time intervals in Fig. $\mathrm{S} 4(\mathrm{a}-\mathrm{d}) \cdot \uparrow$ The $R_{\mathrm{g}}$ of octamers in the two systems also display similar distributions within the two intervals in Fig. S4(e-f). $\dagger$ For comparison, we also calculated the three quantities using the first $100 \mathrm{~ns}$ data and added the results in Fig. $\mathbf{S 4 . \dagger}$ Their distributions (black curves) exhibit significant differences from those within the last 150 ns. Taken together, these results suggest that our REMD simulation for the two systems are reasonably converged after $100 \mathrm{~ns}$. Unless specified, all the REMD simulation results presented below are based on the last $150 \mathrm{~ns}(t=100-250 \mathrm{~ns})$ simulation data generated at $310 \mathrm{~K}$.

\section{Fullerene $\mathrm{C}_{60}$ markedly reduces the $\beta$-sheet content and enhances the coil propensity of GNNQQNY peptide}

The influence of $\mathrm{C}_{60}$ on the secondary structure propensity was monitored by calculating the average probability of each secondary structure (coil, $\beta$-sheet, $\beta$-bridge, bend, turn and helix) over all residues and the residue-based secondary structure probability of GNNQQNY aggregates in the GNN and GNN $+\mathrm{C}_{60}$ 
(a)

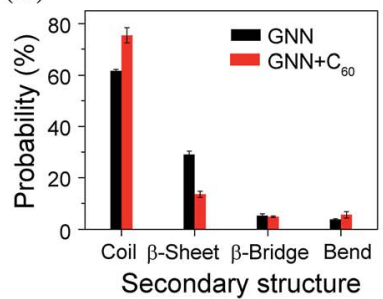

(b)

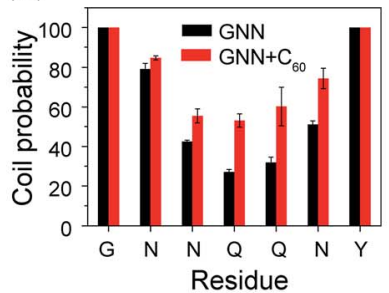

(c)

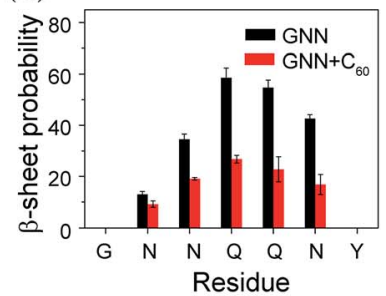

Fig. 1 Secondary structure analysis of GNNQQNY oligomers in GNN and GNN + C $_{60}$ systems. (a) The average probability of each secondary structure (including coil, $\beta$-sheet, $\beta$-bridge and bend) over all residues. The coil (b) and $\beta$-sheet (c) probability as a function of amino acid residue.

systems. As shown in Fig. 1(a), coil and $\beta$-sheet contents are dominant, but both of them are quite different in the two systems. Without $\mathrm{C}_{60}$, the coil and $\beta$-sheet probabilities are $62.7 \%$ and $29.1 \%$, respectively. In the presence of $\mathrm{C}_{60}$, the coil content markedly increases to $75.4 \%$, while the $\beta$-sheet content significantly decreases to $13.5 \%$. The $\beta$-bridge and bend contents are quite similar in the two systems. Note that turn and helix contents $(<1 \%)$ are negligible (data not shown). These results indicate that fullerene $\mathrm{C}_{60}$ can significantly inhibit $\beta$-sheet formation and promote coil formation of hydrophilic GNNQQNY peptide.

Fig. 1(b and c) shows the propensities of coil and $\beta$-sheet as a function of amino acid residue. In the GNN system, residues G1, N2, N3, N6 and Y7 predominantly adopt coil conformations, while middle residues Q4 and Q5 have a high $\beta$-sheet propensity (58.6\% for Q4 and 54.7\% for Q5). We also note that N6 adjacent to $\mathrm{Y} 7$ exhibits a significantly higher $\beta$-sheet probability than other Asn residues (N2 and N3). This observation indicates that $\mathrm{Y} 7$ has a positive effect on the $\beta$-sheet formation of its adjacent residues. In $\mathrm{GNN}+\mathrm{C}_{60}$ system, the introduction of fullerene $\mathrm{C}_{60}$ into GNNQQNY octamers decreases the $\beta$-sheet probability of residues N3, Q4, Q5 and N6 from 34.6-58.6\% to 16.8-26.7\%, while increases the coil percentages from $27.1-42.5 \%$ to $53.1-74.4 \%$. These results demonstrate that $\mathrm{C}_{60}$ nanoparticles can reduce effectively the $\beta$-sheet propensity of residues N3, Q4, Q5 and N6.

GNNQQNY octamers adopt predominantly disordered conformations and to a much lesser extent ordered bilayer $\boldsymbol{\beta}$ sheet structures, while the presence of $\mathrm{C}_{60}$ completely suppresses the ordered bilayer $\beta$-sheets

We further investigated the influence of $\mathrm{C}_{60}$ on the atomic structures of GNNQQNY oligomers. We first calculated the probabilities of different sizes of $\beta$-sheets. As shown in Fig. 3(a), in the GNN system, the majority of the $\beta$-sheets are two-, threeand four-stranded $\beta$-sheets (having a probability of $32.2 \%$, $10.9 \%$ and $9.5 \%$, respectively). In the presence of $\mathrm{C}_{60}$, these three sizes of $\beta$-sheets have a significantly reduced probability (19.8\%, 3.5\% and 3.5\%). Differences are more apparent for larger sizes of $\beta$-sheets. The probabilities of five-, six-, and sevenstranded $\beta$-sheets are respectively $4.9 \%, 1.3 \%$, and $1.2 \%$ in GNN system, while they almost disappear in the $\mathrm{GNN}+\mathrm{C}_{60}$ system.

Experimental studies by Eisenberg et al. showed that $\beta$ strands in GNNQQNY fibrils are in parallel alignment. ${ }^{\mathbf{1 5 , 2 0}}$ It is interesting to examine whether the $\beta$-strands in GNNQQNY oligomers are also in parallel association. To this aim, we calculated the angle between two neighboring strands in all sizes of $\beta$-sheets and the parallel/antiparallel (P/AP) ratio as a function of $\beta$-sheet sizes in GNN system. As shown in Fig. $2(\mathrm{~b})$, the P/AP ratio in two-stranded $\beta$-sheets is quite small (0.04), revealing that strands in two-stranded $\beta$-sheets are mostly in antiparallel association, consistent with previous simulation results. ${ }^{23,24,27,31,35}$ Interestingly, we find that the $\mathrm{P} / \mathrm{AP}$ ratio becomes much larger in $\beta$-sheets with sizes larger than 2 , implying that parallel arrangement of $\beta$-strands becomes favored when $\beta$-sheet sizes increase.

We then examined the structural orderness of GNNQQNY octamers in GNN and GNN $+\mathrm{C}_{60}$ systems by monitoring the time evolution of the connectivity length (CL) (Fig. 2(c)). The CLs of the octamers in the two systems decrease rapidly from the initial value of 8.0 respectively to 6.2 and 7.2 within the first $100 \mathrm{~ns}$ of the simulations and fluctuate around these two values during $t=$ 100-250 ns. The large CL value in the $\mathrm{GNN}+\mathrm{C}_{60}$ system compared to that in GNN system reveals that GNNQQNY octamers become much more disordered in the presence of $\mathrm{C}_{60}$ nanoparticles. (a)

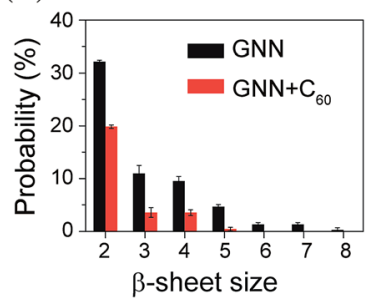

(b)

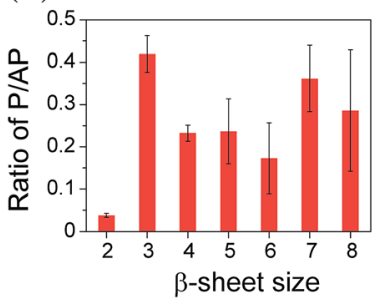

(c)

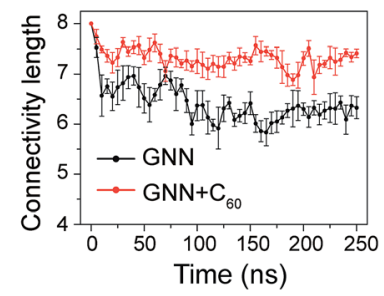

Fig. 2 Analysis of different sizes of $\beta$-sheets and characterization of the structural orderness of GNNQQNY octamers in GNN and GNN + $\mathrm{C}_{60}$ systems. (a) Probability of different sizes of $\beta$-sheet. (b) The P/AP ratio as a function of $\beta$-sheet size in GNN system. (c) The connectivity length as a function of simulation time. 
We also preformed cluster analysis on the GNNQQNY octamers in GNN and GNN $+\mathrm{C}_{60}$ systems. With a $\mathrm{C}_{\alpha}$-RMSD cutoff of $0.3 \mathrm{~nm}$, the GNNQQNY conformations at $310 \mathrm{~K}$ were separated into 139 clusters in GNN system and 150 clusters in $\mathrm{GNN}+\mathrm{C}_{60}$ system. The center conformations of the first eight most-populated cluster are shown in Fig. 3(a) and (b). These clusters represent $46.6 \%$ and $43.5 \%$ of all conformation of GNN and $\mathrm{GNN}+\mathrm{C}_{60}$ systems. Without $\mathrm{C}_{60}$, as shown in Fig. $3(\mathrm{a})$, the first seven clusters are in mixed coil and $\beta$-sheet conformations, while cluster-8 is a bilayer $\beta$-sheet. Both parallel and antiparallel $\beta$-strand alignments are seen in these eight clusters. In the presence of $\mathrm{C}_{60}$, a majority of the eight clusters are in coil-rich conformations (Fig. 3(b)). These results indicate that $\mathrm{C}_{60}$ induces GNNQQNY to form disordered coil-rich aggregates, consistent with the CL analysis shown in Fig. 2(c).

To have an overall view of the conformational distribution of GNNQQNY octamers in the two systems, we constructed in Fig. 4 the potential of mean force as a function of $R_{g}$ and number of H-bonds of GNNQQNY octamers. It is noted that the free energy landscape is a hypersurface function of all the degrees of freedom of the system, while the two-dimensional PMF is a projection of the high-dimensional free energy landscape on two different reaction coordinates. Usually, the PMF is highly degenerate for a complex system involving peptide aggregation ${ }^{35,68,70,86}$ and it cannot discriminate between ordered and disordered conformations. As seen from Fig. 4, the global minimum energy basins of GNNQQNY octamers in the GNN and $\mathrm{GNN}+\mathrm{C}_{60}$ systems are located at $\left(R_{\mathrm{g}}\right.$, number of $\mathrm{H}$-bonds) values of $(0.97 \mathrm{~nm}, 65)$ and $(1.07 \mathrm{~nm}, 55)$, respectively. A decreased number of $\mathrm{H}$-bonds and an increased value of $R_{\mathrm{g}}$ imply that $\mathrm{C}_{60}$ nanoparticles impede the formation of $\mathrm{H}$-bonds and induce the formation of loosely packed aggregates, thus alter the whole free energy surface. We also constructed a PMF as a function of $\beta$-sheet probability and $R_{\mathrm{g}}$ of the octamers (Fig. S5 $\dagger$ ) and found that fullerenes result in a decreased $\beta$-sheet probability and an increased $R_{g}$ of GNNQQNY oligomers.

We also show in Fig. 4 the top four most ordered conformations of GNNQQNY octamers in GNN system and representative disordered coil-rich/ $\beta$-sheet-rich conformations in $\mathrm{GNN}+\mathrm{C}_{60}$ system as well as their locations. In the GNN system, the most ordered clusters are cluster-8, cluster-11, cluster-21 and cluster35. Cluster-8 (3.9\%) and cluster-11 (3.0\%) correspond to bilayer $\beta$-sheet structures, although their $\beta$-strand arrangements are different (see Fig. S6(a-e) $\dagger$ ). Cluster-35 corresponds to $\beta$-barrel structure with a very low probability $(0.5 \%)$. Cluster-21 $(1.3 \%)$ contains loosely packed $\beta$-sheet-rich conformations whose $R_{\mathrm{g}} \mathrm{s}$ are larger than those of bilayer $\beta$-sheets and $\beta$-barrels. For GNN $+\mathrm{C}_{60}$ system, we present four representative disordered clusters: one cluster (cluster-3) corresponding to fully disordered coil conformations and the other three clusters (cluster-5, cluster-51 and cluster-71) corresponding to disordered $\beta$-sheet-rich conformations. The fully disordered coil conformations (cluster-3) are located in the global minimum energy basin. Different sizes of single layer $\beta$-sheets are seen in the disordered $\beta$-sheet-rich conformations, but they are separated by $\mathrm{C}_{60}$ molecules. This finding indicate that $\mathrm{C}_{60}$ molecules play the role as a breaker to block the formation of the fibril-like bi-layer $\beta$-sheet structures.

The GNNQQNY peptides in octamers display a hydration tendency similar to those in fibrils, while the addition of $\mathbf{C}_{60}$ molecules into GNN system increases the water exposure of backbone atoms

Experimental studies of GNNQQNY nanocrystals by Eisenberg et al. using X-ray microcrystallography ${ }^{15}$ and cryo-EM methods ${ }^{20}$ reported that, in the fibril-like nanocrystals, the polar groups of the side chains of residues N2, N3, Q5 and Y7 and the backbone of $\mathrm{G} 1$ and $\mathrm{Y} 7$ are hydrated by water molecules, while the side chains of residues Q4 and N6 are protected from exposing to water molecules. Thus, it is interesting to probe the hydration tendency of these residues in the early formed GNNQQNY oligomers and the influence of fullerenes. To that end, we calculated the number of water molecules within $0.35 \mathrm{~nm}$ from the backbone atoms or side chain atoms of each residue per peptide chain. The side chain of a glycine consists of a single hydrogen atom and this hydrogen atom is included implicitly in the $\mathrm{C}_{\alpha}$ atom in the GROMOS force field. Thus, in the calculation, for residue G1, we considered the number of water molecules contacting with $\mathrm{C}_{\alpha}$ atoms as the number of water molecules that have atomic contacts with the side chains of G1. Fig. 5 shows that, in the absence of fullerenes, the backbone atoms of G1 and Y7 have relatively larger number of contacting water molecules than those of other residues and the side chains of residues N2, Q5 and Y7 are more solvent-exposed than those of other residues. This finding indicates that the relative hydration propensity of amino acid residues of GNNQQNY peptide in early formed octamers is (a) GNN

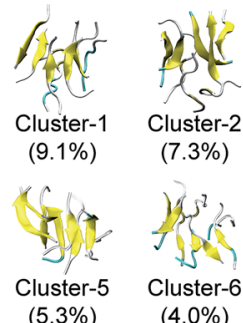

$(4.0 \%)$

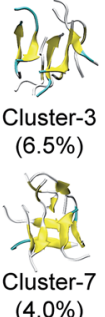

$(4.0 \%)$

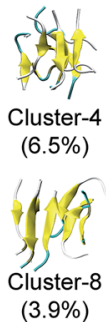

(b) $\mathrm{GNN}+\mathrm{C}_{60}$

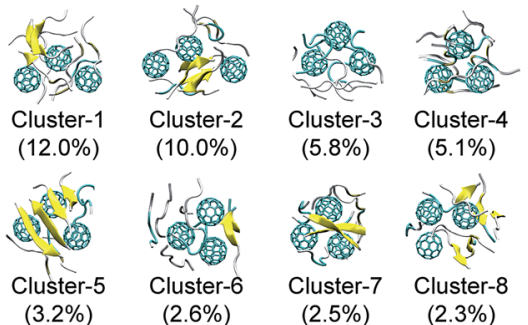

Fig. 3 Representative structures for the first eight most-populated clusters of GNNQQNY octamer alone (a) and GNNQQNY octamer with three $\mathrm{C}_{60}$ molecules (b). 
(a) GNN

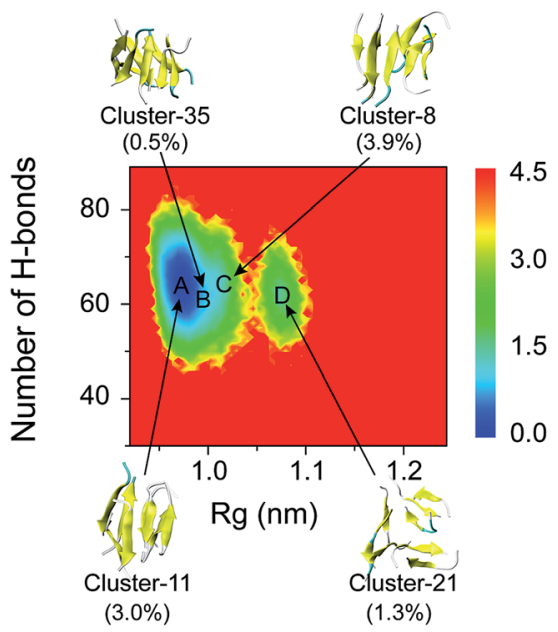

(b) $\mathrm{GNN}+\mathrm{C}_{60}$

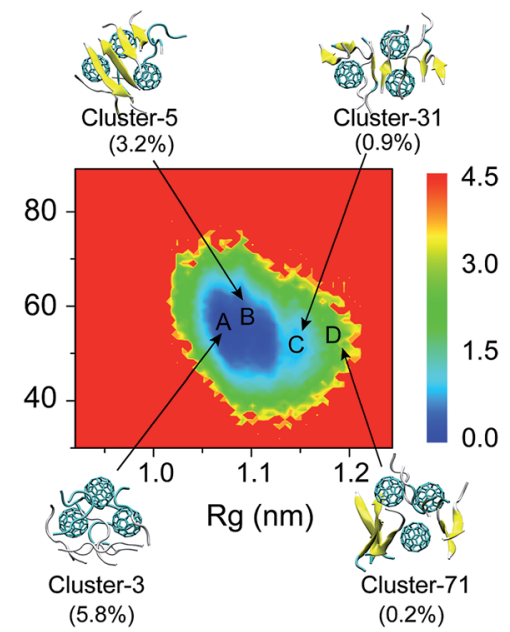

Fig. 4 Potential of mean force (PMF) for GNNQQNY octamers in GNN (a) and GNN + $\mathrm{C}_{60}$ (b) systems. The PMF (in kcal mol ${ }^{-1}$ ) of GNNQQNY octamers plotted as a function of $R_{\mathrm{g}}$ and the number of $\mathrm{H}$-bonds of GNNQQNY octamer. The top four most ordered conformations of octamers in GNN system and representative disordered coil-rich/ $\beta$-sheet-rich conformations in $G N N+C_{60}$ system are also given, along with their probabilities.

(a) Main chain

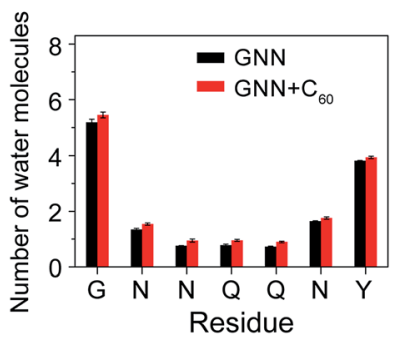

(b) Side chain

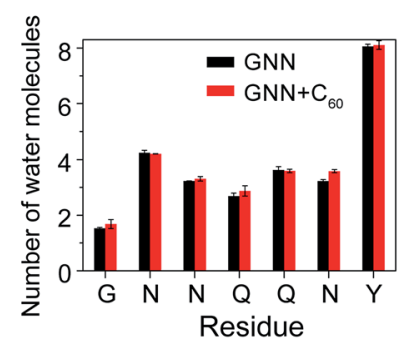

Fig. 5 The number of water molecules within $0.35 \mathrm{~nm}$ of the backbone (a) and side chain (b) atoms of each residue per peptide chain in GNN and GNN + $\mathrm{C}_{60}$ systems.

similar to those in amyloid fibrils, the final products of aggregation, as reported by Eisenberg et al. ${ }^{15,20}$ In the presence of $\mathrm{C}_{60}$ molecules, Fig. 5(a) shows that the number of water molecules around the backbone atoms has a net increase for each residue. Although this net increase looks small for each residue per peptide chain, it would be large when considering all of the eight peptide chains. It can be seen from Fig. 5(b) that for side chain atoms, the number of water molecules around some of the residues increases, while that around other residues decreases, indicating that the number of water molecules around side chain atoms changes slightly. The $\mathrm{C}_{60}$-induced water exposure of peptide backbone atoms would strengthen peptide-water interaction and weaken peptide-peptide interaction, thus interfering with the formation of inter-peptide backbone $\mathrm{H}$-bonds.

\section{Fullerenes block inter-peptide interactions by interacting} strongly with the nonpolar aliphatic groups of polar residues N3, Q4 and Q5

It is of great importance to reveal the binding sites and the physical interactions that fullerenes inhibit the formation of $\beta$ -
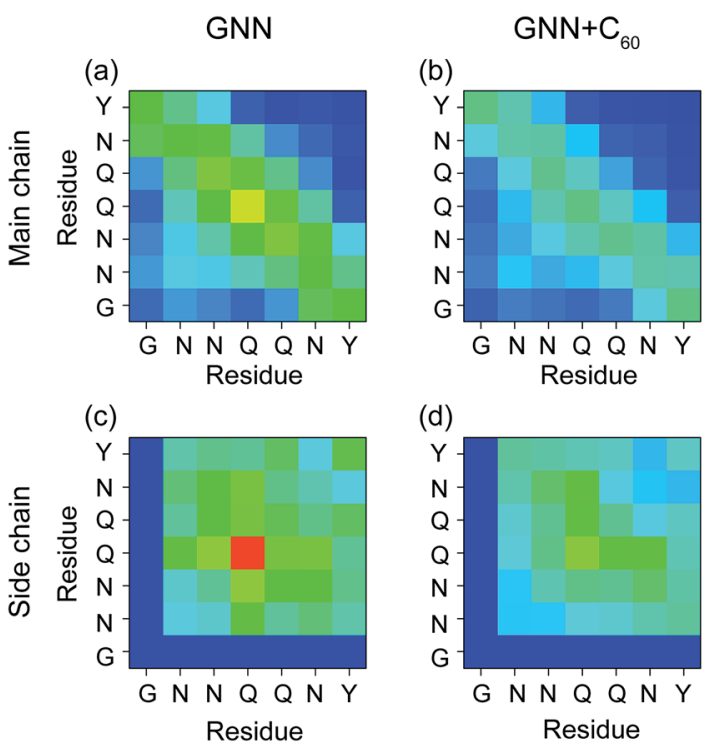

(d)
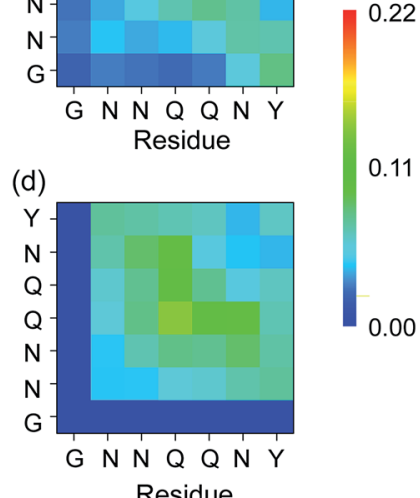

Fig. 6 Inter-peptide MC-MC and SC-SC contact probability maps for GNNQQNY octamers in GNN (a and $c$ ) and GNN + $\mathrm{C}_{60}$ (b and $d$ ) systems.

sheet-rich GNNQQNY octamers. To this aim, we first estimated the inter-peptide main chain-main chain (MC-MC) and side chain-side chain (SC-SC) interactions by calculating the contact probability between each pair of residues. The calculated MC-MC and SC-SC contact probability maps (Fig. 6(a and c)) show that the Q4-Q4 pair has the highest MC-MC and SC-SC contact probabilities, reflecting the important role of $\mathrm{Q} 4$ in the formation of octamers. It can be seen from Fig. 6(a) that residue pairs along the left diagonal of the contact map have relatively high MC-MC contact probabilities, indicating that GNNQQNY peptides are aligned predominantly in antiparallel orientation. 
Interestingly, as shown in Fig. 6(c), the SC-SC contact probability of the $\mathrm{Y} 7-\mathrm{Y} 7$ pair $(9.1 \%)$ is higher than that of the $\mathrm{N} 2-\mathrm{Y} 7$ pair $(6.9 \%)$, implying that GNNQQNY peptides have a propensity to be aligned in a parallel orientation. These data suggest that the orientation between GNNQQNY peptides is modulated by a subtle balance among the aromatic-stacking interactions of the tyrosine rings, the MC-MC and SC-SC interactions of hydrophilic residue pairs. The observation of dominant antiparallel orientation for peptide chains in GNNQQNY octamers elucidates that, in small oligomers, the stacking interaction between the side chains of Y7 residues is less important than the interactions between hydrophilic residue pairs for the alignment orientation of peptide chains, consistent with previous MD simulations. ${ }^{24}$ By comparing Fig. 6(b and d) with (a and c), we find that fullerenes greatly weaken both the MC-MC and SC-SC interactions that are important for GNNQQNY oligomerization.

$\mathrm{H}$-Bonding interactions are considered to be crucial to the fibril formation of the hydrophilic GNNQQNY peptide. ${ }^{21,34,35}$ To identify the key residues for $\mathrm{H}$-bond and $\beta$-sheet formation, we presented in Fig. 7 the MC-MC, SC-SC, and SC-MC H-bond formation maps for GNNQQNY peptides in GNN and GNN + $\mathrm{C}_{60}$ systems. In the GNN system, Fig. 7(a-c) shows that $\mathrm{H}$-bonds are formed between MC-MC, SC-SC and MC-SC atom pairs. The C-terminal residues $\mathrm{Y} 7$ form more $\mathrm{MC}-\mathrm{MC} \mathrm{H}$-bonds with $\mathrm{N}$ terminal residues $\mathrm{N} 2$ and $\mathrm{N} 3$ than with other residues, which facilitates antiparallel $\beta$-strand association. Relatively large number of MC-MC $\mathrm{H}$-bonds are also formed among the middle residues N3, Q4 and Q5, reflecting the important role of those three residues in $\beta$-sheet formation. Interestingly, large number of SC-SC H-bonds are also seen among residues N3, Q4 and Q5. As mentioned above, in the absence of $\mathrm{C}_{60}$, the coil probability is $62.7 \%$ and the majority of octamers are in disordered coil-rich conformations. The formation of large number of SC-SC $\mathrm{H}-$ bonds indicates that inter-peptide SC-SC H-bonding interactions play an important role on the oligomerization of GNNQQNY peptides. However, when $\mathrm{C}_{60}$ nanoparticles were added into the GNN system, both the number of MC-MC and SC-SC H-bonds are greatly reduced, although the $\mathrm{MC}-\mathrm{SC} \mathrm{H}$-bond map is slightly affected. These results imply that $\mathrm{C}_{60}$ molecules markedly impede the formation of inter-peptide $\mathrm{MC}-\mathrm{MC}$ and $\mathrm{SC}-\mathrm{SC} \mathrm{H}-$ bonds which are crucial for both $\beta$-sheet formation and oligomerization of GNNQNY peptides.

To further understand the molecular mechanism that $\mathrm{C}_{60}$ nanoparticles inhibit GNNQQNY oligomerization and $\beta$-sheet formation, we calculated the contact probability and binding free energy between a $\mathrm{C}_{60}$ molecule and each amino acid residue. According to these calculations, we identified the dominant binding sites between $\mathrm{C}_{60}$ molecules and GNNQQNY peptides. As shown in Fig. 8(a), $\mathrm{C}_{60}$ molecules have the highest probabilities $(>30 \%)$ to interact with the hydrophilic residues N3, Q4 and Q5, while have relatively low probability to bind with the aromatic residues Y7. The calculated binding free energy in Fig. 8(b) gives quantitatively the same results as the contact probability does in Fig. 8(a). These data demonstrate that hydrophobic $\mathrm{C}_{60}$ molecules preferentially bind to the middle hydrophilic residues $\mathrm{N} 3$, Q4 and Q5 rather than to the aromatic residues $\mathrm{Y} 7$. The binding modes of $\mathrm{C}_{60}$ molecules to each of the three residues are shown in Fig. 8(c). It can be seen from Fig. 8(c) that $\mathrm{C}_{60}$ molecules bind to the nonpolar aliphatic groups of $\mathrm{N} 3, \mathrm{Q} 4$ and Q5, revealing hydrophobic interactions between $\mathrm{C}_{60}$ and hydrophilic residues, rather than the $\pi-\pi$ stacking interactions between $\mathrm{C}_{60}$ and $\mathrm{Y} 7$, are crucial to the binding of $\mathrm{C}_{60}$ to GNNQQNY peptides. Similarly, strong interactions between carbon nanotubes and hydrophilic residues were reported in previous experimental ${ }^{87}$ and computational $^{88}$ studies on the adsorption of proteins to the surface of carbon nanotubes. As we demonstrated above, inter-peptide SCSC H-bonding interactions among the three residues N3, Q4 and Q5 are crucial to the oligomerization of GNNQQNY peptides. The strong interactions between $\mathrm{C}_{60}$ molecules and these three residues would hinder the inter-peptide interactions responsible for GNNQQNY oligomerization, thus inhibits the fibrillation (a)

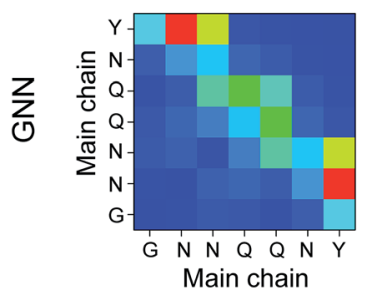

(d)

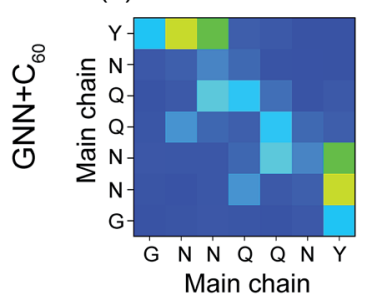

(b)

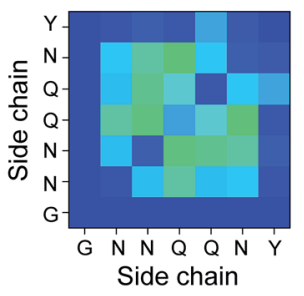

(e)

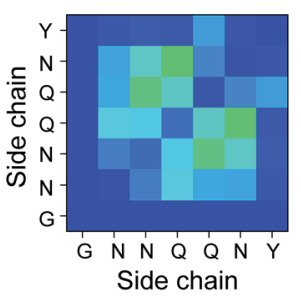

(c)

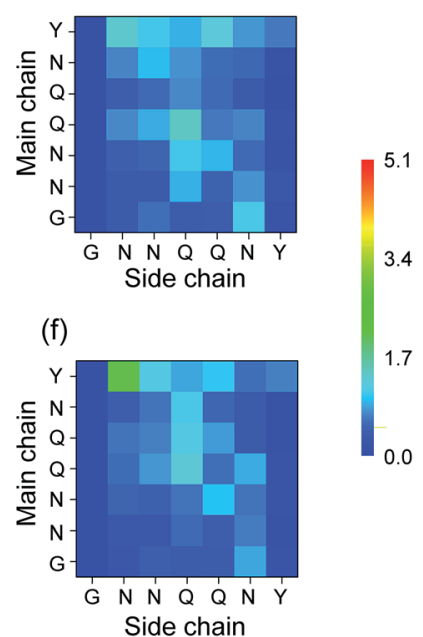

Fig. 7 Inter-peptide $\mathrm{H}$-bond formation maps between each pair of residue in GNN and GNN + $\mathrm{C}_{60}$ systems. MC-MC, SC-SC, and MC-SC Hbond formation maps are shown respectively in ( $a$ and d), (b and e) and (c and f). The number of $\mathrm{H}$-bond is averaged over eight peptide chains. 
(a)

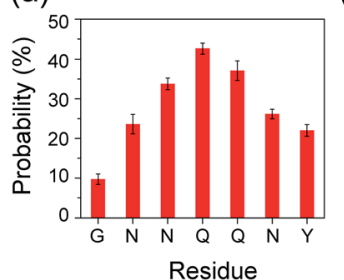

(b)

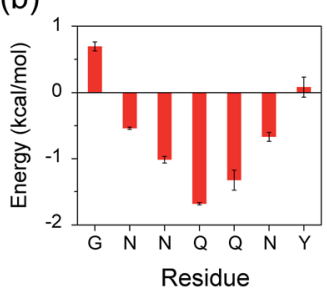

(c)

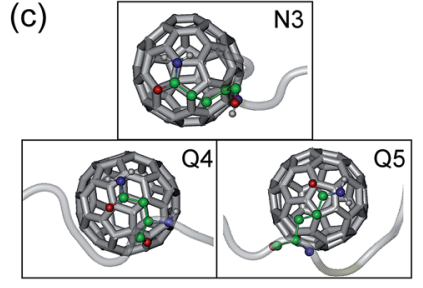

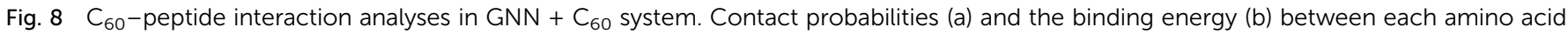

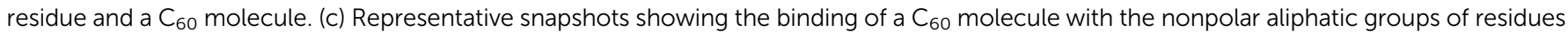

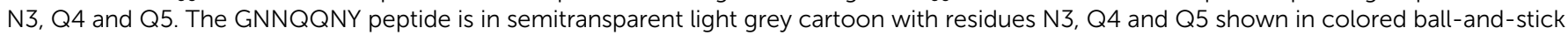
representations to distinguish different type of atoms: $\mathrm{C}$ (green), $\mathrm{N}$ (blue), $\mathrm{O}$ (red), $\mathrm{H}$ (gray). The $\mathrm{C}_{60} \mathrm{molecule}$ is in silver sticks.

process. In our previous REMD studies, we found that carbon nanotubes $^{68}$ and fullerenes ${ }^{50}$ can inhibit effectively the formations of $\beta$-sheet-rich oligomers of the hydrophobic KLVFFAE peptide via hydrophobic and aromatic-stacking interactions and we proposed that carbon nanoparticles may impede the fibrillation of KLVFFAE peptide..$^{\mathbf{5 0 6 8}}$ Interestingly, this prediction was confirmed by atomic force microscopy experiments. ${ }^{\mathbf{5 0 , 7 4}}$ Taken together, we propose that hydrophobic fullerenes could inhibit the aggregation of both hydrophobic and hydrophilic peptides.

\section{Conclusions}

In summary, we investigated the octamerization of GNNQQNY peptide, an amyloid-like fibril-forming fragment of the yeast prion Sup35, and the inhibitory effect of fullerenes on GNNQQNY oligomerization by preforming two $250 \mathrm{~ns}$ atomistic REMD simulations starting from a disordered states. Our simulations show that, in the absence of $\mathrm{C}_{60}$ molecules, GNNQQNY octamers adopt mostly compact disordered conformations including a majority of coil-rich conformations and a minority of $\beta$-sheet-rich conformations. Interestingly, ordered bilayer $\beta$-sheet structures consisting of two fourstranded $\beta$-sheets are also populated, albeit with a low probability $(6.9 \%)$. In the presence of $\mathrm{C}_{60}$ molecules, the coil percentage increases from $62.7 \%$ (without $\mathrm{C}_{60}$ ) to $75.4 \%$, whereas the $\beta$-sheet probability decrease from $29.1 \%$ (without $\mathrm{C}_{60}$ ) to $13.5 \%$. More importantly, $\mathrm{C}_{60}$ molecules greatly enhance the formation of disordered coil-rich conformations, while inhibit $\beta$-sheet-rich conformations and completely suppress ordered fibril-like bilayer $\beta$-sheet structures.

Inter-peptide contact probability maps and $\mathrm{H}$-bond formation maps between all pairs of residues suggest that Q4-Q4 interactions and the SC-SC H-bonding interactions among residues N3, Q4 and Q5 play an important role in the oligomerization of GNNQQNY peptide and the MC-MC H-bonding interactions among residues $\mathrm{N} 3, \mathrm{Q} 4$ and Q5 are crucial for $\beta$ sheet formation. $\mathrm{C}_{60}$ molecules markedly impede the formation of inter-peptide MC-MC and SC-SC H-bonds by binding to the nonpolar aliphatic groups of residues N3, Q4 and Q5 and by exposing peptide backbone atoms to water molecules. The binding energy and binding probability calculation reveals that the hydrophobic interactions between $\mathrm{C}_{60}$ and hydrophilic residues $\mathrm{N} 3, \mathrm{Q} 4$ and $\mathrm{Q} 5$, rather than the $\pi-\pi$ stacking interactions between $\mathrm{C}_{60}$ and residues $\mathrm{Y} 7$, are crucial to the fullerene-peptide interaction. Our computational study suggests that hydrophobic $\mathrm{C}_{60}$ nanoparticles are likely to inhibit the fibrillation of the hydrophilic GNNQQNY peptide.

\section{Acknowledgements}

This work was supported by the NSF of China (Grant No. 11274075 and 11674065) and the MOST of China (Grant No. 2016YFA0501702). Simulations were performed at National High Performance Computing Center of Fudan University.

\section{References}

1 W. Peelaerts, L. Bousset, A. Van der Perren, A. Moskalyuk, R. Pulizzi, M. Giugliano, C. Van den Haute, R. Melki and V. Baekelandt, Nature, 2015, 522, 340-344.

2 F. Chiti and C. M. Dobson, Annu. Rev. Biochem., 2006, 75, 333-366.

3 T. P. Knowles, M. Vendruscolo and C. M. Dobson, Nat. Rev. Mol. Cell Biol., 2014, 15, 384-396.

4 L. C. Serpell, M. D. Benson, G. A. Tennent, M. B. Pepys and P. E. Fraser, J. Mol. Biol., 2000, 300, 1033-1039.

5 D. Thirumalai, D. K. Klimov and R. I. Dima, Curr. Opin. Struct. Biol., 2003, 13, 146-159.

6 D. M. Walsh, I. Klyubin, J. V. Fadeeva, W. K. Cullen, R. Anwyl, M. S. Wolfe, M. J. Rowan and D. J. Selkoe, Nature, 2002, 416, 535-539.

7 R. Kayed, E. Head, J. L. Thompson, T. M. McIntire, S. C. Milton, C. W. Cotman and C. G. Glabe, Science, 2003, 300, 486-489.

8 I. Benilova, E. Karran and B. De Strooper, Nat. Neurosci., 2012, 15, 349-357.

9 J. J. Balbach, Y. Ishii, O. N. Antzutkin, R. D. Leapman, N. W. Rizzo, F. Dyda, J. Reed and R. Tycko, Biochemistry, 2000, 39, 13748-13759.

10 M. von Bergen, P. Friedhoff, J. Biernat, J. Heberle, E. M. Mandelkow and E. Mandelkow, Proc. Natl. Acad. Sci. U. S. A., 2000, 97, 5129-5134.

11 M. I. Ivanova, S. A. Sievers, E. L. Guenther, L. M. Johnson, D. D. Winkler, A. Galaleldeen, M. R. Sawaya, P. J. Hart and D. S. Eisenberg, Proc. Natl. Acad. Sci. U. S. A., 2014, 111, 197-201. 
12 M. Balbirnie, R. Grothe and D. S. Eisenberg, Proc. Natl. Acad. Sci. U. S. A., 2001, 98, 2375-2380.

13 S. L. Hands and A. Wyttenbach, Acta Neuropathol., 2010, 120, 419-437.

14 M. F. Perutz, T. Johnson, M. Suzuki and J. T. Finch, Proc. Natl. Acad. Sci. U. S. A., 1994, 91, 5355-5358.

15 R. Nelson, M. R. Sawaya, M. Balbirnie, A. O. Madsen, C. Riekel, R. Grothe and D. Eisenberg, Nature, 2005, 435, 773-778.

16 P. C. van der Wel, J. R. Lewandowski and R. G. Griffin, J. Am. Chem. Soc., 2007, 129, 5117-5130.

17 K. E. Marshall, M. R. Hicks, T. L. Williams, S. V. Hoffmann, A. Rodger, T. R. Dafforn and L. C. Serpell, Biophys. J., 2010, 98, 330-338.

18 P. C. van der Wel, J. R. Lewandowski and R. G. Griffin, Biochemistry, 2010, 49, 9457-9469.

19 J. R. Lewandowski, P. C. van der Wel, M. Rigney, N. Grigorieff and R. G. Griffin, J. Am. Chem. Soc., 2011, 133, 14686-14698.

20 M. R. Sawaya, J. Rodriguez, D. Cascio, M. J. Collazo, D. Shi, F. E. Reyes, J. Hattne, T. Gonen and D. S. Eisenberg, Proc. Natl. Acad. Sci. U. S. A., 2016, 113, 11232-11236.

21 J. Zheng, B. Ma, C. J. Tsai and R. Nussinov, Biophys. J., 2006, 91, 824-833.

22 L. Esposito, C. Pedone and L. Vitagliano, Proc. Natl. Acad. Sci. U. S. A., 2006, 103, 11533-11538.

23 L. Vitagliano, L. Esposito, C. Pedone and A. De Simone, Biochem. Biophys. Res. Commun., 2008, 377, 1036-1041.

24 Z. Zhang, H. Chen, H. Bai and L. Lai, Biophys. J., 2007, 93, 1484-1492.

25 J. Wang, C. Tan, H. F. Chen and R. Luo, Biophys. J., 2008, 95, 5037-5047.

26 A. S. Reddy, M. Chopra and J. J. de Pablo, Biophys. J., 2010, 98, 1038-1045.

27 J. Nasica-Labouze, M. Meli, P. Derreumaux, G. Colombo and N. Mousseau, PLoS Comput. Biol., 2011, 7, e1002051.

28 J. Nasica-Labouze and N. Mousseau, PLoS Comput. Biol., 2012, 8, e1002782.

29 B. Barz, D. J. Wales and B. Strodel, J. Phys. Chem. B, 2014, 118, 1003-1011.

30 Y. Sugita, A. Kitao and Y. Okamoto, J. Chem. Phys., 2000, 113, 6042-6051.

31 A. Srivastava and P. V. Balaji, J. Struct. Biol., 2015, 192, 376391.

32 G. Reddy, J. E. Straub and D. Thirumalai, Proc. Natl. Acad. Sci. U. S. A., 2009, 106, 11948-11953.

33 D. Thirumalai, G. Reddy and J. E. Straub, Acc. Chem. Res., 2012, 45, 83-92.

34 J. Gsponer, U. Haberthur and A. Caflisch, Proc. Natl. Acad. Sci. U. S. A., 2003, 100, 5154-5159.

35 B. W. Strodel, C. S. Whittleston and D. J. Wales, J. Am. Chem. Soc., 2007, 16005-16014.

36 K. L. Osborne, M. Bachmann and B. Strodel, Proteins, 2013, 81, 1141-1155.

37 C. Cabaleiro-Lago, F. Quinlan-Pluck, I. Lynch, S. Lindman, A. M. Minogue, E. Thulin, D. M. Walsh, K. A. Dawson and S. Linse, J. Am. Chem. Soc., 2008, 130, 15437-15443.

38 Y. H. Liao, Y. J. Chang, Y. Yoshiike, Y. C. Chang and Y. R. Chen, Small, 2012, 8, 3631-3639.
39 M. Mahmoudi, O. Akhavan, M. Ghavami, F. Rezaee and S. M. Ghiasi, Nanoscale, 2012, 4, 7322-7325.

40 T. Takahashi and H. Mihara, Acc. Chem. Res., 2008, 41, 13091318.

41 B. Cheng, H. Gong, H. Xiao, R. B. Petersen, L. Zheng and K. Huang, Biochim. Biophys. Acta, 2013, 1830, 4860-4871.

42 D. E. Ehrnhoefer, J. Bieschke, A. Boeddrich, M. Herbst, L. Masino, R. Lurz, S. Engemann, A. Pastore and E. E. Wanker, Nat. Struct. Mol. Biol., 2008, 15, 558-566.

43 Q. Wang, X. Yu, K. Patal, R. Hu, S. Chuang, G. Zhang and J. Zheng, ACS Chem. Neurosci., 2013, 4, 1004-1015.

44 F. De Leo, A. Magistrato and D. Bonifazi, Chem. Soc. Rev., 2015, 44, 6916-6953.

45 D. Lin, R. Qi, S. Li, R. He, P. Li, G. Wei and X. Yang, ACS Chem. Neurosci., 2016, 7, 1232-1240.

46 M. Zhang, X. Mao, Y. Yu, C. X. Wang, Y. L. Yang and C. Wang, Adv. Mater., 2013, 25, 3780-3801.

47 C. Li and R. Mezzenga, Nanoscale, 2013, 5, 6207-6218.

48 L. Wang, S. Zhu, T. Lu, G. Zhang, J. Xu, Y. Song, Y. Li, L. Wang, B. Yang and F. Li, J. Mater. Chem. B, 2016, 4, 4913-4921.

49 S. H. Friedman, D. L. DeCamp, R. P. Sijbesma, G. Srdanov, F. Wudl and G. L. Kenyon, J. Am. Chem. Soc., 1993, 115, 6506-6509.

50 L. Xie, Y. Luo, D. Lin, W. Xi, X. Yang and G. Wei, Nanoscale, 2014, 6, 9752-9762.

51 L. L. Dugan, D. M. Turetsky, C. Du, D. Lobner, M. Wheeler, C. R. Almli, C. K. Shen, T. Y. Luh, D. W. Choi and T. S. Lin, Proc. Natl. Acad. Sci. U. S. A., 1997, 94, 9434-9439.

52 J. E. Kim and M. Lee, Biochem. Biophys. Res. Commun., 2003, 303, 576-579.

53 Y. Ishida, T. Fujii, K. Oka, D. Takahashi and K. Toshima, Chem.-Asian J., 2011, 6, 2312-2315.

54 A. G. Bobylev, A. B. Kornev, L. G. Bobyleva, M. D. Shpagina, I. S. Fadeeva, R. S. Fadeev, D. G. Deryabin, J. Balzarini, P. A. Troshin and Z. A. Podlubnaya, Org. Biomol. Chem., 2011, 9, 5714-5719.

55 E. G. Makarova, R. Y. Gordon and I. Y. Podolski, J. Nanosci. Nanotechnol., 2012, 12, 119-126.

56 V. Vorobyov, V. Kaptsov, R. Gordon, E. Makarova, I. Podolski and F. Sengpiel, JAD, J. Alzheimer's Dis., 2015, 45, 217-233.

57 Z. Bednarikova, P. D. Huy, M. M. Mocanu, D. Fedunova, M. S. Li and Z. Gazova, Phys. Chem. Chem. Phys., 2016, 18, 18855-18867.

58 S. A. Andujar, F. Lugli, S. Hofinger, R. D. Enriz and F. Zerbetto, Phys. Chem. Chem. Phys., 2012, 14, 8599-8607.

59 X. Zhou, W. Xi, Y. Luo, S. Cao and G. Wei, J. Phys. Chem. B, 2014, 118, 6733-6741.

60 P. D. Huy and M. S. Li, Phys. Chem. Chem. Phys., 2014, 16, 20030-20040.

61 Y. Sun, Z. Qian and G. Wei, Phys. Chem. Chem. Phys., 2016, 18, 12582-12591.

62 M. Haratake, T. Takiguchi, N. Masuda, S. Yoshida, T. Fuchigami and M. Nakayama, Colloids Surf., B, 2016, 149, 72-79.

63 P. C. van der Wel, J. R. Lewandowski and R. G. Griffin, J. Am. Chem. Soc., 2007, 129, 5117-5130. 
64 Y. Sugita and Y. Okamoto, Chem. Phys. Lett., 1999, 314, 141151.

65 D. Van Der Spoel, E. Lindahl, B. Hess, G. Groenhof, A. E. Mark and H. J. Berendsen, J. Comput. Chem., 2005, 26, 1701-1718.

66 R. Qi, Y. Luo, B. Ma, R. Nussinov and G. Wei, Biomacromolecules, 2014, 15, 122-131.

67 J. Lei, R. Qi, G. Wei, R. Nussinov and B. Ma, Phys. Chem. Chem. Phys., 2016, 18, 8098-8107.

68 H. Li, Y. Luo, P. Derreumaux and G. Wei, Biophys. J., 2011, 101, 2267-2276.

69 W. F. van Gunsteren, S. R. Billeter, A. A. Eising, P. H. Hünenberger, P. Krüger, A. E. Mark, W. R. P. Scott and I. G. Tironi, Biomolecular Simulation: The GROMOS96 Manual and User Guide, Vdf Hochschulverlag AG an der ETH Zürich, Zürich, Switzerland, 1996, pp. 1-1042.

70 Z. A. Levine, L. Larini, N. E. LaPointe, S. C. Feinstein and J. E. Shea, Proc. Natl. Acad. Sci. U. S. A., 2015, 112, 2758-2763.

71 A. Morriss-Andrews and J. E. Shea, Annu. Rev. Phys. Chem., 2015, 66, 643-666.

72 J. Nasica-Labouze, P. H. Nguyen, F. Sterpone, O. Berthoumieu, N. V. Buchete, S. Cote, A. De Simone, A. J. Doig, P. Faller, A. Garcia, A. Laio, M. S. Li, S. Melchionna, N. Mousseau, Y. Mu, A. Paravastu, S. Pasquali, D. J. Rosenman, B. Strodel, B. Tarus, J. H. Viles, T. Zhang, C. Wang and P. Derreumaux, Chem. Rev., 2015, 115, 3518-3563.

73 Y. Zou, Y. Sun, Y. Zhu, B. Ma, R. Nussinov and Q. Zhang, ACS Chem. Neurosci., 2016, 7, 286-296.
74 L. Xie, D. Lin, Y. Luo, H. Li, X. Yang and G. Wei, Biophys. J., 2014, 107, 1930-1938.

75 T. Darden, D. York and L. Pedersen, J. Chem. Phys., 1993, 98, 10089.

76 B. Hess, H. Bekker, H. J. Berendsen and J. G. E. M. Fraaije, J. Comput. Chem., 1997, 18, 1463-1472.

77 S. Miyamoto and P. A. Kollman, J. Comput. Chem., 1992, 13, 952-962.

78 G. Bussi, D. Donadio and M. Parrinello, J. Chem. Phys., 2007, 126, 014101.

79 M. Parrinello and A. Rahman, Phys. Rev. Lett., 1980, 45, 1196-1199.

80 Y. Lu, P. Derreumaux, Z. Guo, N. Mousseau and G. Wei, Proteins: Struct., Funct., Bioinf., 2009, 75, 954-963.

81 X. Daura, K. Gademann, B. Jaun, D. Seebach, W. F. van Gunsteren and A. E. Mark, Angew. Chem., Int. Ed., 1999, 38, 236-240.

82 R. Kumari, R. Kumar, C. Open Source Drug Discovery and A. Lynn, J. Chem. Inf. Model., 2014, 54, 1951-1962.

83 N. Homeyer and H. Gohlke, Mol. Inf., 2012, 31, 114-122.

84 W. M. Berhanu and U. H. Hansmann, Proteins, 2013, 81, 1542-1555.

85 W. Humphrey, A. Dalke and K. Schulten, J. Mol. Graphics, 1996, 14, 33-38.

86 W. Song, G. Wei, N. Mousseau and P. Derreumaux, J. Phys. Chem. B, 2008, 112, 4410-4418.

87 S. Brown, T. S. Jespersen and J. Nygard, Small, 2008, 4, 416420.

88 G. Zuo, S. G. Kang, P. Xiu, Y. Zhao and R. Zhou, Small, 2013, 9, 1546-1556. 